\title{
AN ALGEBRAIC REPRESENTATION OF GLOBULAR SETS
}

\author{
A. M. MEDINA-MARDONES
}

\begin{abstract}
We describe a fully faithful embedding of the category of (reflexive) globular sets into the category of counital cosymmetric $R$-coalgebras when $R$ is an integral domain. This embedding is a lift of the usual functor of $R$-chains and the extra structure consists of a derived form of cup coproduct. Additionally, we construct a functor from group-like counital cosymmetric $R$-coalgebras to $\omega$-categories and use it to connect two fundamental constructions associated to oriented simplices: Steenrod's cup- $i$ coproducts and Street's orientals. The first defines the square operations in the cohomology of spaces, the second, the nerve of higher-dimensional categories.
\end{abstract}

\section{INTRODUCTION}

Globular sets are presheaves over a category $\mathbb{G}$ whose objects are non-negative integers. They generalize directed graphs and constitute one of the major geometric shapes for higher category theory, providing models for strict and non-strict higher-dimensional categories when enriched with further structure.

We depict the representable globular set $\mathbb{G}_{n}$ for small values of $n$ :

$$
n=0 \quad n=1
$$
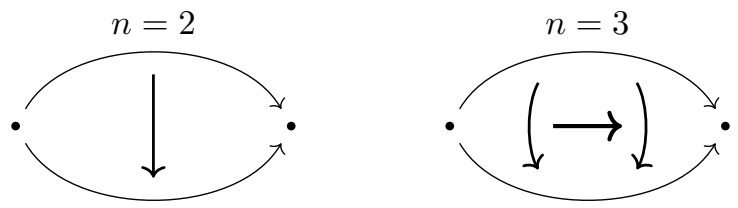

The globular set $\partial \mathbb{G}_{n+1}$ obtained by removing the identity from $\mathbb{G}_{n+1}$ models the $n$-sphere together with its antipodal map. We are interested in the functor C. of chains from globular sets to differential graded $R$-modules. Let $W$ be defined as the colimit of the diagram

$$
\text { C. }\left(\partial \mathbb{G}_{0}\right) \rightarrow \mathrm{C} \bullet\left(\partial \mathbb{G}_{1}\right) \rightarrow \cdots
$$

induced from a standard set of inclusions $\mathbb{G}_{n} \rightarrow \mathbb{G}_{n+1}$. We notice that the antipodal map makes $W$ into a free differential graded $R\left[\Sigma_{2}\right]$-module. For any globular set $X$ we will construct a natural $R\left[\Sigma_{2}\right]$-module chain map

$$
\Delta: W \otimes \mathrm{C}_{\bullet}(X) \rightarrow \mathrm{C} \bullet(X) \otimes \mathrm{C}_{\bullet}(X)
$$

together with a natural chain map $\varepsilon: \mathrm{C} .(X) \rightarrow R$ satisfying appropriate counitality relations. We can think of this structure as a lift to the chain level of the counital cocommutative $R$-coalgebra on the homology of $X$ (a structure pre-dual to the usual cup product in cohomology).

We will show that when $R$ is an integral domain, this lift of the functor of chains is a fully faithful embedding of the category of globular sets into the category of counital cosymmetric $R$-coalgebras. We can think of this result as a non-linear globular form of the Dold-Kan Theorem. In more diagramatic language,

Key words and phrases. globular sets, higher categories, $E_{\infty}$-structures, Steenrod cup- $i$ products. 
our map fits into the following commutative diagram

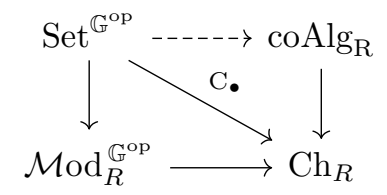

where the lower triangle consists of a free functor followed by a fully faithful embedding and the upper triangle consists of a fully faithful embedding followed by a forgetful functor.

We will then focus on the full subcategory $\operatorname{coAlg}_{R}^{g l}$ of group-like counital cosymmetric $R$-coalgebras and on a model for strict higher-dimensional categories known as $\omega$-categories. We will describe a functor, similar to those used by Street, Brown, and Steiner in their respective studies of parity complexes, linear $\omega$-categories, and augmented directed complexes, from $\operatorname{coAlg}_{R}^{g l}$ to $\omega$ Cat behaving like a free functor on pasting diagrams. We will use our version to relate two fundamental constructions on oriented simplices: Steenrod's cup- $i$ coproducts and Street's orientals. The first defines the square operations on the cohomology of spaces, the second, the nerve of $\omega$-categories.

Acknowledgments. We would like to thank Chris Schommer-Pries, Tim Campion, Stephan Stolz, Dennis Sullivan, Richard Steiner, Manuel Rivera, Mahmoud Zeinalian, and the anonymous referee for their insights, questions, and comments about this project.

\section{Globular Sets and Counital Cosymmetric $R$-COAlgebras}

In this section we will describe how to represent, when $R$ is an integral domain, the category of globular sets algebraically as a full subcategory of the category of counital cosymmetric $R$-coalgebras. These are models for counital $R$-coalgebras commutative up to coherent homotopies ( $E_{\infty}$-coalgebras are examples). We will also review an important construction of Steenrod providing concrete examples of such $R$-coalgebra when $R=\mathbb{F}_{2}$ and used to define his square operations.

2.1. Globular sets. The globe category $\mathbb{G}$ has set of objects the non-negative integers and its morphisms are generated by

subject to the relations

$$
\sigma_{n}, \tau_{n}: n \rightarrow n+1 \quad \iota_{n}: n \rightarrow n-1
$$

$$
\begin{aligned}
\tau_{n} \tau_{n-1} & =\sigma_{n} \tau_{n-1} & \sigma_{n} \sigma_{n-1} & =\tau_{n} \sigma_{n-1} \\
\iota_{n+1} \tau_{n} & =\mathrm{id}_{n} & \iota_{n+1} \sigma_{n} & =\mathrm{id}_{n} .
\end{aligned}
$$

Let Set be the category of small sets. We denote the category of contravariant functors from $\mathbb{G}$ to Set by Set $^{\mathbb{G}^{\text {op }}}$ and refer to it as the category of globular sets. For a globular set $X$ we use the notation

$$
X_{n}=X(n) \quad t_{n}=X\left(\tau_{n}\right) \quad s_{n}=X\left(\sigma_{n}\right) \quad i_{n}=X\left(\iota_{n}\right) .
$$

Furthermore, abusing notation, we let $t_{n}: X(k) \rightarrow X(n)$ stand for any composition of the form $t_{n} r$ where $r: X(k) \rightarrow X(n+1)$ is induced from an arbitrary morphism. Thanks to (11) this map is independent of $r$ and determined by the integer $k$. We follow a similar convention for $s_{n}$.

2.2. Augmented differential graded $R$-modules. Let $R$ be a commutative and unital ring. The category of differential (homologically) graded $R$-modules concentrated in non-negative degrees is denoted $\mathrm{Ch}_{R}$. We reserve the word chain complex for when $R$ equals $\mathbb{Z}$.

Let $C$ be a differential graded $R$-module and $n$ a non-negative integer, we denote

$$
C_{\leq n}=C_{0} \oplus C_{1} \oplus \cdots \oplus C_{n} .
$$

A pair $(C, \varepsilon)$ with $C$ and $\varepsilon: C \rightarrow R$ in $\mathrm{Ch}_{R}$ is called an augmented differential graded $R$-module and a morphism between two of them is a morphism of underlying differential graded $R$-modules making the diagram 


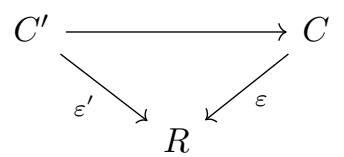

commutative.

The functor C. : Set ${ }^{\mathbb{G}^{o p}} \rightarrow \mathrm{Ch}_{R}$ is defined for $X \in \operatorname{Set}^{\mathbb{G}^{\text {op }}}$ by

$$
\mathrm{C}_{n}(X)=R\left\{X_{n}\right\} / R\left\{i_{n}\left(X_{n-1}\right)\right\} \quad \partial_{n}=t_{n-1}-s_{n-1} .
$$

It admits a natural lift to the category of augmented differential graded $R$-modules by defining for $x \in X_{n}$

$$
\varepsilon(x)= \begin{cases}1 & n=0 \\ 0 & n \neq 0 .\end{cases}
$$

2.3. Counital cosymmetric $R$-coalgebras. Let $\Sigma_{2}$ be the group with one non-identity element $T$. Let us consider the following resolution of $R$ by free $R\left[\Sigma_{2}\right]$-modules:

$$
W=R\left[\Sigma_{2}\right] \stackrel{1-T}{\longleftarrow} R\left[\Sigma_{2}\right] \stackrel{1+T}{\longleftarrow} R\left[\Sigma_{2}\right] \stackrel{1-T}{\longleftarrow} \ldots
$$

and let $\varepsilon_{W}: W \rightarrow R$ be the unique $R\left[\Sigma_{2}\right]$-linear map extending the identity $R \rightarrow R$.

Given any differential graded $R$-module $C$ we make $C \otimes C$ into a differential graded $R\left[\Sigma_{2}\right]$-module using the transposition of factors $T(x \otimes y)=(-1)^{r s} y \otimes x$ where $r$ and $s$ are the degrees of $x$ and $y$.

A counital cosymmetric $R$-coalgebra is an augmented differential graded $R$-module $(C, \varepsilon)$ together with

$$
\Delta: W \otimes C \rightarrow C \otimes C
$$

an $R\left[\Sigma_{2}\right]$-linear chain map making the following diagrams commute:
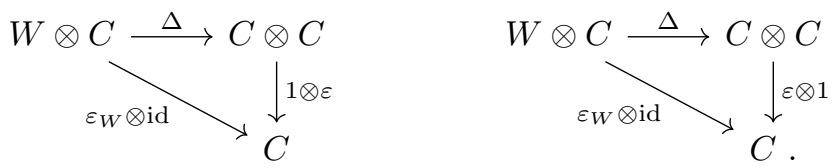

A coalgebra map between counital cosymmetric $R$-coalgebras is a map $f$ of underlying augmented differential graded $R$-modules making the following diagram commute:

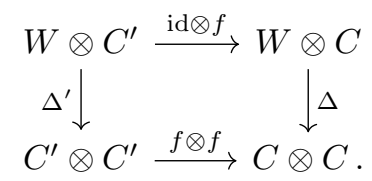

We denote the category of counital cosymmetric $R$-coalgebras with coalgebra maps by $\operatorname{coAlg}_{R}$.

We use the adjunction isomorphism

$$
\operatorname{Hom}_{R\left[\Sigma_{2}\right]}(W \otimes C, C \otimes C) \rightarrow \operatorname{Hom}_{R\left[\Sigma_{2}\right]}(W, \operatorname{Hom}(C, C \otimes C))
$$

to represent $\Delta$ by a collection of maps $\Delta_{k}: C \rightarrow C \otimes C$ satisfying

$$
\partial \Delta_{k}-(-1)^{k} \Delta_{k} \partial=\left(1+(-1)^{k} T\right) \Delta_{k-1}
$$

with the convention that $\Delta_{-1}=0$.

2.4. Steenrod cup- $i$ coalgebras. Alexander-Whitney's approximation to the diagonal map

$$
\Delta_{0}: \mathrm{C} \bullet \rightarrow \mathrm{C} \bullet \otimes \mathrm{C} \bullet
$$

defines a natural non-cocommutative coproduct on the integral chains of any simplicial set whose linear dual descends to the commutative cup product on its cohomology. In Ste47, Steenrod constructed a cosymmetric $\mathbb{Z}$-coalgebra

$$
\Delta: W \otimes \mathrm{C}_{\bullet} \rightarrow \mathrm{C} \bullet \otimes \mathrm{C}_{\bullet}
$$

extending the Alexander-Whitney coproduct, which when considered with $\mathbb{F}_{2}$-coefficients defines the square operations

$$
S q^{k}: H^{\bullet}\left(-; \mathbb{F}_{2}\right) \rightarrow H^{\bullet+k}\left(-; \mathbb{F}_{2}\right) .
$$


Since these operations are homological in nature, any pair of natural homotopy equivalent cosymmetric $\mathbb{F}_{2^{-}}$ coalgebra structures give rise to isomorphic square operations. Yet, Steenrod's original construction appears ubiquitously in the literature in various equivalent forms. For example, in MM18c, the author finds it in the action of a finitely presented prop arising from just three maps: Alexander-Whitney's diagonal, the augmentation, and the join map. In MM18b, it is induced from the action of a cellular $E_{\infty}$-operad on the geometric realization of cubical sets. And in MS03] and BF04, McClure-Smith and Berger-Fresse find it in the action of their respective Sequence and Barratt-Eccles operads.

The universality of this cosymmetric $\mathbb{F}_{2}$-coalgebra is formalized via an axiomatic characterization in MM18a. In this note, we provide further evidence for its fundamental nature by deriving from it in Theorem 17 another fundamental construction: the nerve of higher-dimensional categories.

Let us review its description as presented in MM18a. Let $P\left(\begin{array}{l}n \\ k\end{array}\right)$ be the set of all $U=\left\{0 \leq u_{1}<\cdots<\right.$ $\left.u_{k} \leq n\right\}$. For any such $U$ define the composition of face maps

$$
d_{U}=d_{u_{1}} \cdots d_{u_{k}}
$$

and the pair

$$
\begin{aligned}
& U^{-}=\left\{u_{i} \in U: u_{i} \not \equiv i \bmod 2\right\} \\
& U^{+}=\left\{u_{i} \in U: u_{i} \equiv i \bmod 2\right\} .
\end{aligned}
$$

Definition 1. (MM18a $)$ For any simplicial set $X$ its Steenrod cup- $i$ coalgebra $\left(\mathrm{C} \bullet\left(X ; \mathbb{F}_{2}\right), \Delta, \varepsilon\right)$ is defined by

$$
\Delta_{i}(x)=\sum_{U \in P\left(\begin{array}{c}
n \\
n-i
\end{array}\right)} d_{U^{-}} x \otimes d_{U^{+}} x
$$

and

where $x \in X_{n}$.

$$
\varepsilon(x)= \begin{cases}1 & n=0 \\ 0 & n \neq 0\end{cases}
$$

Remark 2. The cup product and Steenrod squares in cohomology are obtained from the Steenrod cup- $i$ coalgebra by defining

$$
[\alpha] \smile[\beta]=\left[(\alpha \otimes \beta) \Delta_{0}\right]
$$

and

$$
S q^{k}[\alpha]=\left[(\alpha \otimes \alpha) \Delta_{|\alpha|-k}\right]
$$

The relationship between these two cohomological structures is known as the Cartan Formula. In [MM19, using the definitions above, the author gave an effective chain level proof of the Cartan Formula, and in [MM18d], based on (1), a novel algorithm for the computation of Steenrod squares of finite simplicial complexes was developed and added to the field of topological data analysis.

2.5. Globular $R$-coalgebras. We now describe a counital cosymmetric $R$-coalgebra naturally associated to a globular set and state our main theorem.

Definition 3. For any globular set $X$ its globular $R$-coalgebra $(\mathrm{C} \bullet(X ; R), \Delta, \varepsilon)$ is defined by

$$
\Delta_{k}(x)= \begin{cases}0 & n<k \\ x \otimes x & n=k \\ t_{k} x \otimes x+(-1)^{(n+1) k} x \otimes s_{k} x & k<n\end{cases}
$$

and

where $x \in X_{n}$.

$$
\varepsilon(x)= \begin{cases}1 & n=0 \\ 0 & n>0\end{cases}
$$


Theorem 4. Let $R$ be an integral domain. The assignment

$$
X \rightarrow(\mathrm{C} \bullet(X ; R), \Delta, \varepsilon)
$$

induces a full and faithful embedding of $\operatorname{Set}^{\mathbb{G}^{\circ \mathrm{p}}}$ into $\operatorname{coAlg}_{R}$.

The proof of this theorem occupies Section 4.

Remark 5. We can think of this statement as a non-linear globular form of the Dold-Kan Theorem. A conjecture, verified in the author's thesis [Med15] for special cases, is that including the higher arity parts of an $E_{\infty}$-coalgebra structure on the chains of simplicial sets results in a similar non-linear (simplicial) Dold-Kan Theorem.

\section{Group-Like COALGEBras AND higher-Dimensional CATEGORIES}

3.1. $\omega$-categories and the functor $\mu$. In this subsection we recall the definition of $\omega$-categories, which are a globular model of strict higher-dimensional categories. We also review a natural construction associating an $\omega$-category to any differential graded $R$-module.

Definition 6. An $\omega$-category is a globular set $X$ together with maps

$$
\circ_{m}: X_{n} \times_{X_{m}} X_{n} \rightarrow X_{m}
$$

where

$$
X_{n} \times_{X_{m}} X_{n}=\{(y, x) \in X \times X \mid s(y)=t(x)\}
$$

satisfying relations of associativity, unitality and interchange. For the complete list of relations we refer the reader to Definition 1.4.8 in Lei04. When $t_{m}(x)=s_{m}(y)=z$ we write $y \circ_{z} x$ for $y \circ_{m} x$.

The next definition appears in [Ste04] where is partially credited to [BH03] and [Str91].

Definition 7 (Street, Brown-Higgins, Steiner). The functor

$$
\mu: \mathrm{Ch}_{R} \rightarrow \omega \mathrm{Cat}
$$

is defined as follows: for $C$ a differential graded $R$-module let $\mu(C)$ be the $R$-submodule of the infinite product of $C$ with itself generated by all sequences

$$
c=\left(c_{0}^{-}, c_{0}^{+}, c_{1}^{-}, c_{1}^{+}, \ldots\right)
$$

satisfying

i) $c_{n}^{-}, c_{n}^{+} \in C_{n}$

ii) $c_{n}^{-}, c_{n}^{+}=0$ for $n>>0$,

iii) $\partial c_{n+1}^{-}=\partial c_{n+1}^{+}=c_{n}^{+}-c_{n}^{-}$.

We can make this $R$-module into a globular set by defining

$$
\mu(C)_{n}=\left\{c \in \mu(C): \forall k>n, c_{k}^{-}=c_{k}^{+}=0\right\}
$$

and

$$
\begin{aligned}
s_{k}(c) & =\left(c_{0}^{-}, c_{0}^{+}, \ldots, c_{k-1}^{-}, c_{k-1}^{+}, c_{k}^{-}, c_{k}^{-}, 0,0, \ldots\right) \\
t_{k}(c) & =\left(c_{0}^{-}, c_{0}^{+}, \ldots, c_{k-1}^{-}, c_{k-1}^{+}, c_{k}^{+}, c_{k}^{+}, 0,0, \ldots\right) \\
i_{k}(c) & =c .
\end{aligned}
$$

We can make this globular set into an $\omega$-category by defining

$$
\begin{aligned}
b \circ_{c} a & =b+a-c \\
& =\left(b_{0}^{-}+a_{0}^{-}-c_{0}^{-}, b_{0}^{+}+a_{0}^{+}-c_{0}^{+}, \ldots\right) .
\end{aligned}
$$


3.2. Group-like coalgebras and the functor $\xi$. In this subsection we define group-like elements in counital cosymmetric $R$-coalgebras and consider $\operatorname{coAlg}_{R}^{g l}$, the full subcategory of counital cosymmetric $R$ coalgebras admitting a basis of group-like elements. We then introduce a functor from $\operatorname{coAlg}_{R}^{g l}$ to $\omega$ Cat using the notion of atom associated to a group-like element.

Definition 8. Let $(C, \Delta, \varepsilon)$ be a counital cosymmetric coalgebra. We call $c \in C_{n}$ a group-like element if for any integer $k$ we have

$$
\begin{gathered}
\Delta_{k}(c) \in C_{\leq n} \otimes C_{\leq n} \\
\Delta_{n}(c)=c \otimes c
\end{gathered}
$$

and, when $n=0$,

$$
\varepsilon(c)=1 \text {. }
$$

We say that $C$ is group-like if it admits a basis of group-like elements and denote the full subcategory of group-like counital cosymmetric $R$-coalgebras as $\operatorname{coAlg}_{R}^{g l}$.

A consequence of the following lemma applied to the identity map is that if a counital cosymmetric coalgebra admits a basis of group-like elements, then that basis is unique.

Lemma 9. Let $R$ be an integral domain. If $f: R[A] \rightarrow R[B]$ is a coalgebra map between counital cosymmetric $R$-coalgebras with bases of group-like elements $A$ and $B$. Then, for any $a \in A$ either $f(a)=0$ or there exists $b \in B$ such that $f(a)=b$.

Proof. For $a \in A_{n}$ there is a collection of elements $b_{i} \in B_{n}$ and coefficients $\beta_{i} \in R$ such that

$$
f(a)=\sum_{i} \beta_{i} b_{i}
$$

Applying $\Delta_{n}$ to (4) gives

$$
\sum_{i} \beta_{i} b_{i} \otimes b_{i}=\Delta_{n} f(a)=(f \otimes f) \Delta_{n}(a)=\sum_{i, j} \beta_{i} \beta_{j} b_{i} \otimes b_{j} .
$$

The equations $0=\beta_{i} \beta_{j}$ for $i \neq j$ together with $\beta_{i}=\beta_{i}^{2}$ imply, since $R$ is an integral domain, that each coefficient $\beta_{i}$ equals 0 except possibly one of them that must equal 1 .

Example 10. Steenrod cup- $i$ coalgebras as well as globular $R$-coalgebras are group-like.

Definition 11. Let $C$ be a differential graded $R$-module with a basis $B$. For $b \in B$ let $\pi_{b}: C \rightarrow R$ be the $R$-linear map sending $b$ to 1 and the other basis elements to 0 . Define the maps

$$
\pi_{b}^{+}, \pi_{b}^{-}: C \otimes C \rightarrow C
$$

by

where $T$ is the transposition of factors.

$$
\pi_{b}^{+}=\mathrm{id} \otimes \pi_{b} \quad \text { and } \quad \pi_{b}^{-}=\pi_{b}^{+} T
$$

We make a note of the following straightforward observation for later use:

Lemma 12. Let $C$ be a differential graded $R$-module with a basis $B$. If $b \in B_{n}$ and $\eta \in\{+,-\}$ then

on $C_{\leq n} \otimes C_{\leq n}$ and

$$
\partial \pi_{b}^{\eta}=\pi_{b}^{\eta} \partial
$$

on $C_{\leq n-1} \otimes C_{\leq n-1}$.

$$
\pi_{b}^{\eta}=0
$$

Definition 13. Let $(C, \Delta, \varepsilon)$ be a counital cosymmetric coalgebra. For every group-like element $b \in C$ define its atom as

with

$$
\langle b\rangle=\left(\langle b\rangle_{0}^{-},\langle b\rangle_{0}^{+},\langle b\rangle_{1}^{-},\langle b\rangle_{1}^{+}, \ldots\right)
$$

$$
\langle b\rangle_{k}^{\eta}= \begin{cases}(-1)^{k} \pi_{b}^{-} \Delta_{k} b & \eta=- \\ \pi_{b}^{+} \Delta_{k} b & \eta=+.\end{cases}
$$


Lemma 14. Let $C$ be a counital cosymmetric coalgebra. For any group-like element $b \in C$ the atom $\langle b\rangle$ is in $\mu(C)$.

Proof. We need to prove that for any group-like element $b$ of degree $n$ the sequence $\langle b\rangle$ satisfies conditions i), ii), and iii) in Definition 7 Since the first two conditions are immediate, we are left with showing that for any non-negative integer $k$

$$
\partial\langle b\rangle_{k+1}^{+}=\partial\langle b\rangle_{k+1}^{-}=\langle b\rangle_{k}^{+}-\langle b\rangle_{k}^{-}
$$

For $k>n$ and $\eta \in\{+,-\}$ we have $\langle b\rangle_{k}^{\eta}=0$ so (5) holds. For $k=n$ we notice that $\langle b\rangle_{k}^{+}=\langle b\rangle_{k}^{-}=b$ and (5) follows. For $k<n$, using Lemma 12, we have

$$
\begin{aligned}
\langle b\rangle_{k}^{+}-\langle b\rangle_{k}^{-} & =\pi_{b}^{+} \Delta_{k} b-(-1)^{k} \pi_{b}^{-} \Delta_{k} b=\pi_{b}^{+}\left(1+(-1)^{k+1} T\right) \Delta_{k} b \\
& =\pi_{b}^{+}\left(\partial \Delta_{k+1} b-(-1)^{k} \Delta_{k+1} \partial b\right)=\partial \pi_{b}^{+} \Delta_{k+1} b \\
& =\partial\langle b\rangle_{k+1}^{+}
\end{aligned}
$$

and

$$
\begin{aligned}
\langle b\rangle_{k}^{+}-\langle b\rangle_{k}^{-} & =\pi_{b}^{+} \Delta_{k} b-(-1)^{k} \pi_{b}^{-} \Delta_{k} b=\pi_{b}^{-}\left(T+(-1)^{k+1}\right) \Delta_{k} b \\
& =(-1)^{k+1} \pi_{b}^{-}\left(\partial \Delta_{k+1} b-(-1)^{k} \Delta_{k+1} \partial b\right) \\
& =(-1)^{k+1} \partial \pi_{b}^{-}\left(\Delta_{k+1} b\right)=\partial\langle b\rangle_{k+1}^{-}
\end{aligned}
$$

as desired.

Lemma 15. Let $R$ be an integral domain. The assignment sending a group-like counital cosymmetric $R$ coalgebra $C$ to the sub- $\omega$-category of $\mu(C)$ generated by its atoms is functorial.

Proof. The statement follows from the fact, proven in Lemma 9, that when $R$ is an integral domain a coalgebra map between group-like $R$-coalgebras sends group-like elements to either group-like elements or to 0 .

\section{Definition 16. Let}

be the functor described in Lemma 15

$$
\xi: \operatorname{coAlg}_{R}^{g l} \rightarrow \omega \mathrm{Cat}
$$

3.3. Street's orientals. In this subsection we state the second main result of this note: the functor $\xi$ sends the Steenrod coalgebra of a standard simplex to the free $\omega$-category generated by that simplex.

Historically, Roberts Rob77, pioneered the idea of using higher-dimensional categories as the coefficient objects for non-abelian cohomology. A key ingredient for this enterprise is the construction of a nerve functor from $\omega$-categories to simplicial sets. Such a functor $N$ can be obtained from the construction of a natural cosimplicial $\omega$-category

$$
\begin{aligned}
\mathcal{O}: \Delta & \rightarrow \omega \text { Cat } \\
{[n] } & \mapsto \mathcal{O}_{n}
\end{aligned}
$$

by setting

$$
N(\mathcal{C})_{n}=\operatorname{Hom}_{\omega \mathrm{Cat}}\left(\mathcal{O}_{n}, \mathcal{C}\right) .
$$

This was accomplished by Street in [Str87] where he says the following about the $\omega$-categories $\mathcal{O}_{n}$ : "[t] hese objects seem to be fundamental structures of nature so I decided they should have a short descriptive name. I settled on oriental."

We will not use the original definition of Street but an equivalent one given by Steiner in Ste04 and further explored in [Ste07. It is presented as Definition 20 after a review of Steiner's theory of augmented directed complexes.

We are ready to state the second main result of this work.

Theorem 17. Let $\left(\mathrm{C} \cdot\left(\Delta^{n} ; \mathbb{F}_{2}\right), \Delta, \varepsilon\right)$ be the Steenrod cup-i coalgebra associated to the $n$-th representable simplicial set $\boldsymbol{\Delta}^{n}$. Then,

$$
\xi\left(\mathrm{C} \bullet\left(\boldsymbol{\Delta}^{n} ; \mathbb{F}_{2}\right), \Delta, \varepsilon\right)=\mathcal{O}_{n} .
$$


The proof of this theorem occupies subsection 3.5 .

3.4. Steiner's augmented directed complexes. In this section we give an extremely abridged exposition of Steiner's rich theory of augmented directed complexes with the aim of proving Theorem 17. The original source is [Ste04].

We refer to the objects of $\mathrm{Ch}_{\mathbb{Z}}$ simply as chain complexes.

Let $C$ be a chain complex together with a basis. We write $C^{+}$for the submonoid containing all elements written as linear combinations of basis elements with only non-negative coefficients. We use the following notation for the induced canonical decomposition:

$$
c=c^{+}-c^{-}
$$

with $c^{+}$and $c^{-}$in $C^{+}$.

Let $(C, \varepsilon)$ be an augmented chain complex with a basis $B$. For $b \in B_{n}$ define recursively

$$
b_{i}^{+}=\left\{\begin{array}{ll}
0 & i>n \\
b & i=n \\
\left(\partial b_{i+1}^{+}\right)^{+} & i<n
\end{array} \quad \text { and } \quad b_{i}^{-}= \begin{cases}0 & i>n \\
b & i=n \\
\left(\partial b_{i+1}^{-}\right)^{-} & i<n .\end{cases}\right.
$$

The basis is said to be unital if $\varepsilon\left(b_{0}^{+}\right)=\varepsilon\left(b_{0}^{-}\right)=1$ for every $b \in B$.

Definition 18. A strong augmented directed complex or simply a SADC is an augmented chain complex $C$ with a unital basis such that the transitive closure of the reflexive relation $\leq$ defined by

$$
c_{1} \leq c_{2}
$$

if and only if

$$
\left(\partial c_{2}\right)^{-}-c_{1} \in C^{+}
$$

or

$$
\left(\partial c_{1}\right)^{+}-c_{2} \in C^{+}
$$

is anti-symmetric, i.e., it defines a partial order on $C$.

A morphism between two SADCs is an augmented chain map $f: C_{1} \rightarrow C_{2}$ such that

$$
f\left(C_{1}^{+}\right) \subset f\left(C_{2}^{+}\right) .
$$

Definition 19. Let $(C, B)$ be a SADC. For $b \in B$ define its Steiner atom to be

$$
\left(b_{0}^{-}, b_{0}^{+}, b_{1}^{-}, b_{1}^{+}, \ldots\right) \in \mu(C) .
$$

Steiner showed that assigning to a SADC, let us call it $(C, B)$, the sub- $\omega$-category generated inside $\mu(C)$ by its Steiner atoms defines a full and faithful embedding

$$
\nu: \mathrm{SADC} \rightarrow \omega \mathrm{Cat}
$$

independent of the commutative and unital $\operatorname{ring} R$ used in the construction of $\mu$. We refer the reader to 5.6, 6.1 , and 6.2 in Ste04 for these statements.

Additionally, Steiner gives the following definition of Street's orientals in 3.8 loc. cit.:

Definition 20 ([Ste04]). Let $\boldsymbol{\Delta}^{n}$ denote the $n$-th representable simplicial set. The chain complex C. $\left(\boldsymbol{\Delta}^{n} ; \mathbb{Z}\right)$ together with the canonical basis

$$
B=\{[m] \rightarrow[n]: \text { injective }\}
$$

define a SADC and

$$
\mathcal{O}_{n}=\nu\left(\mathrm{C}_{\bullet}\left(\boldsymbol{\Delta}^{n} ; \mathbb{Z}\right), B\right)
$$


3.5. Proof of Theorem 17. We will exhibit a bijection between the set of atoms of $\xi\left(\mathrm{C} \bullet\left(\boldsymbol{\Delta}^{n} ; \mathbb{F}_{2}\right), \Delta, \varepsilon\right)$ and of Steiner atoms of $\nu\left(\mathrm{C}_{\bullet}\left(\boldsymbol{\Delta}^{n} ; \mathbb{Z}\right), B\right)$ which, since these are generators, will establish the theorem.

We will verify that for every non-degenerate simplex $\sigma:[m] \rightarrow[n]$ and $\eta \in\{-,+\}$ we have

$$
\sigma_{i}^{\eta}=\pi_{\sigma}^{\eta} \Delta_{i} \sigma
$$

where this equality holds with $\mathbb{Z}$-coefficients using the canonical set lift $\mathbb{F}_{2} \rightarrow \mathbb{Z}$ with $0 \mapsto 0$ and $1 \mapsto 1$.

For $i>m$, both sides of (7) are equal to 0 .

For $i \leq m$, let $r=m-i$. Then, by (3), we have

$$
\pi_{\sigma}^{-} \Delta_{i} \sigma=\sum_{\substack{U \in P\left(\begin{array}{c}
m \\
r
\end{array}\right) \\
U^{-}=\emptyset}} d_{U^{+}} \sigma \quad \text { and } \quad \pi_{\sigma}^{+} \Delta_{i} \sigma=\sum_{\substack{U \in P\left(\begin{array}{c}
m \\
r
\end{array}\right) \\
U^{+}=\emptyset}} d_{U^{-}} \sigma .
$$

Then, in the case $r=0$, (77) holds because $\pi_{\sigma}^{-} \Delta_{i} \sigma=\sigma$ and $\pi_{\sigma}^{+} \Delta_{i} \sigma=\sigma$, so $\pi_{\sigma}^{-} \Delta_{i} \sigma=\sigma_{i}^{-}$and $\pi_{\sigma}^{+} \Delta_{i} \sigma=\sigma_{i}^{+}$. Assuming the identity for $r$ we compute

$$
\partial \sigma_{i}^{-}=\sum_{j}(-1)^{j} d_{j} \sigma_{i}^{-}=\sum_{j}(-1)^{j} \sum_{\substack{U \in P\left(\begin{array}{c}
m \\
r
\end{array}\right) \\
U^{-}=\emptyset}} d_{j} d_{U^{+}} \sigma .
$$

We will prove the identity for $r+1$ by rewriting the above identity as

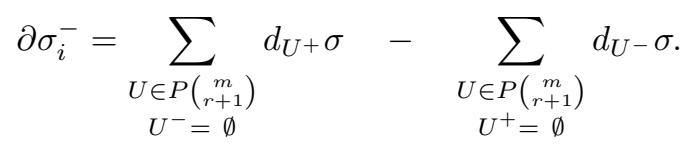

For $U=\left\{u_{1}<\cdots<u_{r}\right\} \in P\left(\begin{array}{l}n \\ r\end{array}\right)$ with $U^{-}=\emptyset$ and $0 \leq j \leq i$ we can use the simplicial identities to write

$$
d_{j} d_{U^{+}}=d_{j} d_{u_{1}} \ldots d_{u_{r}}=d_{u_{1}} \ldots d_{u_{l}} d_{j+l} d_{u_{l}+1} \ldots d_{u_{r}}
$$

with $u_{l}<j+l<u_{l+1}$. Notice that if $j \equiv 1 \bmod 2$ and $l<r$ then

$$
V=\left\{u_{1}<\cdots<u_{l}<j+l<\widehat{u}_{l+1}<\cdots<u_{r}\right\} \in P\left(\begin{array}{c}
m \\
r
\end{array}\right)
$$

with $V^{-}=\emptyset$ and, calling $k=u_{l+1}-l-1$,

$$
(-1)^{j} d_{j} d_{U}+(-1)^{k} d_{k} d_{V}=0 .
$$

If $j \equiv 0 \bmod 2$ and $1<l$ then

$$
W=\left\{u_{1}<\cdots<\widehat{u}_{l}<j+l<u_{l+1}<\cdots<u_{r}\right\} \in P\left(\begin{array}{c}
m \\
r
\end{array}\right)
$$

with $W^{-}=\emptyset$ and, calling $k=u_{l}-l$,

$$
(-1)^{j} d_{j} d_{U}+(-1)^{k} d_{k} d_{W}=0 .
$$

This implies that the only non-zero terms are of the form

$$
\begin{cases}d_{u_{1}} \ldots d_{u_{r}} d_{j+r} & j \text { odd } \\ d_{j} d_{u_{1}} \ldots d_{u_{r}} & j \text { even }\end{cases}
$$

for $U=\left\{u_{1}<\cdots<u_{r}\right\} \in P\left(\begin{array}{l}n \\ r\end{array}\right)$ with $U^{-}=\emptyset$. Therefore,

$$
\partial \sigma_{i}=\sum_{\substack{U \in P\left(\begin{array}{c}
m \\
r+1
\end{array}\right) \\
U^{-}=\emptyset}} d_{U^{+}} \sigma-\sum_{\substack{U \in P\left(\begin{array}{c}
m \\
r+1
\end{array}\right) \\
U^{+}=\emptyset}} d_{U^{-}} \sigma
$$

as claimed. 


\section{Proof of Theorem 4}

We will prove Theorem 4 by establishing a sequence of lemmas. Unless stated otherwise, all algebraic constructions are taken over a general commutative and unital ring $R$.

Lemma 21. For any globular set $X$ the triple $(\mathrm{C} \bullet(X), \Delta, \varepsilon)$ is a counital cosymmetric $R$-coalgebras.

Proof. Showing that $\Delta: W \otimes \mathrm{C}_{\bullet}(X) \rightarrow \mathrm{C} \bullet(X) \otimes \mathrm{C}_{\bullet}(X)$ is a $R\left[\Sigma_{2}\right]$-linear chain map is equivalent to establishing (2) for all $k \geq 0$. We will split the verification into six cases. For the remainder of this proof let us consider $x \in X_{n}$.

If $k=n=0$ :

$$
\begin{aligned}
\partial \Delta_{k} x-(-1)^{k} \Delta_{k} \partial x & =\partial(x \otimes x) \\
& =0 .
\end{aligned}
$$

If $k=0<n$ :

$$
\begin{aligned}
\partial \Delta_{k} x-(-1)^{k} \Delta_{k} \partial x & =t_{0} x \otimes\left(t_{n-1}-s_{n-1}\right) x+\left(t_{n-1}-s_{n-1}\right) x \otimes s_{0} x \\
& -t_{0} t_{n-1} x \otimes t_{n-1} x-t_{n-1} x \otimes s_{0} t_{n-1} x \\
& +t_{0} s_{n-1} x \otimes s_{n-1} x+t_{n-1} x \otimes s_{0} s_{n-1} x \\
& =0 .
\end{aligned}
$$

If $0<k=n+1$ :

$$
\begin{aligned}
\partial \Delta_{k} x-(-1)^{k} \Delta_{k} \partial x & =0 \\
& =x \otimes x-x \otimes x \\
& =\left(1+(-1)^{n+1} T\right)(x \otimes x) \\
& =\left(1+(-1)^{k} T\right) \Delta_{k-1}(x) .
\end{aligned}
$$

If $0<k=n$ :

$$
\begin{aligned}
\partial \Delta_{k} x-(-1)^{k} \Delta_{k} \partial x & =\left(t_{n-1}-s_{n-1}\right) x \otimes x+(-1)^{n} x \otimes\left(t_{n-1}-s_{n-1}\right) x \\
& =\left(t_{k-1} x \otimes x-(-1)^{n} x \otimes s_{k-1} x\right) \\
& +(-1)^{n}\left(x \otimes t_{k-1} x-(-1)^{n} s_{k-1} x \otimes x\right) \\
& =\left(1+(-1)^{n} T\right)\left(t_{k-1} x \otimes x-(-1)^{n} x \otimes s_{k-1} x\right) \\
& =\left(1+(-1)^{n} T\right)\left(t_{k-1} x \otimes x+(-1)^{(n+1)(k-1)} x \otimes s_{k-1} x\right) \\
& =\left(1+(-1)^{n} T\right) \Delta_{k-1}(x) .
\end{aligned}
$$

If $0<k=n-1$ :

$$
\begin{aligned}
\partial \Delta_{k} x-(-1)^{k} \Delta_{k} \partial x & =\left(t_{k-1}-s_{k-1}\right) t_{k} x \otimes x+(-1)^{(n-1)} t_{k} x \otimes\left(t_{n-1}-s_{n-1}\right) x \\
& +(-1)^{n+1}\left(t_{n-1}-s_{n-1}\right) x \otimes s_{k} x-x \otimes\left(t_{k-1}-s_{k-1}\right) s_{k} x \\
& +(-1)^{k+1}\left(t_{k} x \otimes t_{k} x-s_{k} x \otimes s_{k} x\right) \\
& =\left(t_{k-1} x \otimes x+x \otimes s_{k-1} x\right)-\left(x \otimes t_{k-1} x+s_{k-1} x \otimes x\right) \\
& =\left(1+(-1)^{k} T\right)\left(t_{k-1} x \otimes x+x \otimes s_{k-1} x\right) \\
& =\left(1+(-1)^{k} T\right) \Delta_{k-1}(x)
\end{aligned}
$$


If $0<k<n-1$ :

$$
\begin{aligned}
\partial \Delta_{k} x-(-1)^{k} \Delta_{k} \partial x & =\left(t_{k-1}-s_{k-1}\right) t_{k} x \otimes x+(-1)^{k} t_{k} x \otimes\left(t_{n-1}-s_{n-1}\right) x \\
& +(-1)^{(n+1) k}\left(\left(t_{n-1}-s_{n-1}\right) x \otimes s_{k} x+(-1)^{n} x \otimes\left(t_{k-1}-s_{k-1}\right) s_{k} x\right) \\
& +(-1)^{k+1}\left(t_{k} t_{n-1} x \otimes t_{n-1} x+(-1)^{n k} t_{n-1} x \otimes s_{k} t_{n-1} x\right) \\
& +(-1)^{k}\left(t_{k} s_{n-1} x \otimes s_{n-1} x+(-1)^{n k} s_{n-1} x \otimes s_{k} s_{n-1} x\right) \\
& =\left(t_{k-1} x \otimes x+(-1)^{(n+1)(k+1)} x \otimes s_{k-1} x\right) \\
& +\left((-1)^{(n(k+1)+k)} x \otimes t_{k-1} x-s_{k-1} x \otimes x\right) \\
& =\left(1+(-1)^{k} T\right)\left(t_{k-1} x \otimes x+(-1)^{(n+1)(k-1)} x \otimes s_{k-1} x\right) \\
& =\left(1+(-1)^{k} T\right) \Delta_{k-1}(x) .
\end{aligned}
$$

Showing that $\varepsilon$ is a counit for $\Delta$ follows from the fact that for any $x \in X_{n}$

$$
\Delta_{0} x= \begin{cases}t_{0} x \otimes x+x \otimes s_{0} x & n \neq 0 \\ x \otimes x & n=0\end{cases}
$$

and $\varepsilon\left(x^{\prime}\right)=1$ for any $x^{\prime} \in X_{0}$.

Lemma 22. For any morphism $F: X \rightarrow Y$ of globular sets, the chain map

$$
\mathrm{C} .(F): \mathrm{C} .(X) \rightarrow \mathrm{C} \bullet(Y)
$$

is a coalgebra map.

Proof. Denote C. $(F)$ by $f$. Since $F\left(X_{n}\right) \subseteq Y_{n}$ we have $\varepsilon f=f \varepsilon$ and since $F t_{n}=t_{n} F$ and $F s_{n}=s_{n} F$ we have

$$
\begin{aligned}
(f \otimes f) \Delta_{k}(x) & =F\left(t_{k} x\right) \otimes F(x)+(-1)^{(n+1) k} F(x) \otimes F\left(s_{k} x\right) \\
& =t_{k} F(x) \otimes F(x)+(-1)^{(n+1) k} F(x) \otimes s_{k} F(x) \\
& =\Delta_{k} f(x)
\end{aligned}
$$

for any $x \in X$ and $k \geq 0$.

Lemma 23. If $R$ is an integral domain, the function

$$
\operatorname{Set}^{\mathbb{G}^{\mathrm{op}}}(X, Y) \rightarrow \operatorname{coAlg}_{R}(\mathrm{C} \bullet(X), \mathrm{C} \bullet(Y))
$$

is a bijection.

Proof. Injectivity is immediate. For establishing surjectivity, let us consider $f \in \operatorname{coAlg}_{R}\left(\mathrm{C}_{\bullet}(X), \mathrm{C}_{\bullet}(Y)\right)$. We will construct $F \in \operatorname{Set}^{\mathbb{G}^{\text {op }}}(X, Y)$ such that $\mathrm{C} \cdot(F)=f$. From Lemma 9 we know that for any $x \in X$ either $f(x)=y$ for some $y \in Y$ or $f(x)=0$. Let $x \in X_{n}$ not in the image of $i_{n}$. Define

$$
F(x)= \begin{cases}f(x) & f(x) \neq 0 \\ i_{n}\left(F\left(t_{n-1} x\right)\right) & f(x)=0 .\end{cases}
$$

This recursive definition is well defined because of the augmentation preserving property of $f$. For $x=i_{n}(y)$ we recursively define $F(x)=i_{n} F(y)$. We will prove next that $F: X \rightarrow Y$ is a map of globular sets.

Let $x \in X_{n}$ and without loss of generality assume it is not in the image of $i_{n}$. Let us first assume $f(x)=0$, then

$$
t_{n-1} F(x)=t_{n-1} i_{n} F\left(t_{n-1} x\right)=F\left(t_{n-1} x\right)
$$

and

$$
s_{n-1} F(x)=s_{n-1} i_{n} F\left(t_{n-1} x\right)=F\left(t_{n-1} x\right) \stackrel{?}{=} F\left(s_{n-1} x\right) .
$$

We claim that $F\left(t_{n-1} x\right)$ must equal $F\left(s_{n-1} x\right)$. Observe that since $f$ is a chain map

$$
f(x)=0 \Rightarrow f\left(t_{n-1} x\right)=f\left(s_{n-1} x\right)
$$


If $f\left(t_{n-1} x\right) \neq 0$ or $f\left(s_{n-1} x\right) \neq 0$ implication (8) establishes the claims. If $f\left(t_{n-1} x\right)=f\left(s_{n-1} x\right)=0$ we have

$$
F\left(t_{n-1} x\right)=i_{n-1} F\left(t_{n-2} t_{n-1} x\right)=i_{n-1} F\left(t_{n-2} s_{n-1} x\right)=F\left(s_{n-1} x\right) .
$$

Let us now assume $f(x) \neq 0$. For any $0<k \leq n$ the identity

$$
\Delta_{n-k} f(x)=(f \otimes f) \Delta_{n-k}(x)
$$

reads

$$
\begin{aligned}
t_{n-k} F(x) \otimes F(x) & +(-1)^{(n+1) k} F(x) \otimes s_{n-k} F(x) \\
& =f\left(t_{n-k} x\right) \otimes F(x)+(-1)^{(n+1) k} F(x) \otimes f\left(s_{n-k} x\right) .
\end{aligned}
$$

Therefore, for $0<k \leq n$

$$
\begin{aligned}
f\left(t_{n-k} x\right) \neq 0 & \Rightarrow t_{n-k} F(x)=F\left(t_{n-k} x\right) \\
f\left(s_{n-k} x\right) \neq 0 & \Rightarrow s_{n-k} F(x)=F\left(s_{n-k} x\right)
\end{aligned}
$$

Therefore, if both $f\left(t_{n-1} x\right) \neq 0$ and $f\left(s_{n-1} x\right) \neq 0$ we are done.

Let us assume $f\left(t_{n-1} x\right)=0$ and notice that $n-1$ must be greater than 0 . It follows from (9) that $t_{n-1} F(x)$ is in the image of $i_{n-1}$. Writing $t_{n-1} F(x)=i_{n-1} y$ and applying $t_{n-2}$ to this identity gives $t_{n-2} F(x)=y$. Hence,

$$
F\left(t_{n-1} x\right) \stackrel{\text { def }}{=} i_{n-1} F\left(t_{n-2} t_{n-1} x\right)=i_{n-1} F\left(t_{n-2} x\right) \stackrel{?}{=} i_{n-1} t_{n-2} F(x)=i_{n-1} y=t_{n-1} F(x) .
$$

Similarly, when $f\left(s_{n-1} x\right)=0$ we have

$$
F\left(s_{n-1} x\right) \stackrel{\text { def }}{=} i_{n-1} F\left(t_{n-2} s_{n-1} x\right)=i_{n-1} F\left(t_{n-2} x\right) \stackrel{?}{=} i_{n-1} t_{n-2} F(x)=s_{n-1} F(x) .
$$

Therefore, we have reduced both claims: $F\left(t_{n-1} x\right)=t_{n-1} F(x)$ when $f\left(t_{n-1} x\right)=0$ and $s_{n-1} F(x)=$ $F\left(s_{n-1} x\right)$ when $f\left(s_{n-1} x\right)=0$ to showing $F\left(t_{n-2} x\right)=t_{n-2} F(x)$. If $f\left(t_{n-2} x\right) \neq 0$ then (10) finishes the proof. If not, we repeat the argument and reduce it to $F\left(t_{n-3} x\right)=t_{n-3} F(x)$. Because of the augmentation preserving property of $f$ this regression has to end.

\section{REFERENCES}

[BF04] Clemens Berger and Benoit Fresse. Combinatorial operad actions on cochains. In Mathematical Proceedings of the Cambridge Philosophical Society, volume 137, pages 135-174. Cambridge University Press, 2004.

[BH03] Ronald Brown and Philip J Higgins. Cubical abelian groups with connections are equivalent to chain complexes. Homology, Homotopy and Applications, 5(1):49-52, 2003.

[Lei04] Tom Leinster. Higher operads, higher categories, volume 298. Cambridge University Press, 2004.

[Med15] Anibal Medina. $E_{\infty}$-Comodules and Topological Manifolds. PhD thesis, Stony Brook University, 2015.

[MM18a] A. M. Medina-Mardones. An axiomatic characterization of Steenrod's cup- $i$ products. arXiv preprint arXiv:1810.06505, 2018.

[MM18b] A. M. Medina-Mardones. A finitely presented $E_{\infty}$-prop II: Cellular context. arXiv preprint arXiv:1808.07132, 2018.

[MM18c] A. M. Medina-Mardones. A finitely presented $E_{\infty}$-prop I: Differential graded context. arXiv preprint arXiv:1808.00854, 2018.

[MM18d] A. M. Medina-Mardones. Persistence Steenrod modules. arXiv preprint arXiv:1812.05031, 2018.

[MM19] A. M. Medina-Mardones. An effective proof of the Cartan Formula. arXiv preprint arXiv:1907.12113, 2019.

[MS03] James McClure and Jeffrey Smith. Multivariable cochain operations and little n-cubes. Journal of the American Mathematical Society, 16(3):681-704, 2003.

[Rob77] John E Roberts. Mathematical aspects of local cohomology. In Algebres dopérateurs et leurs applications en physique mathématique.(Proc. Colloq. Marseille, 1977), pages 321-332, 1977.

[Ste47] Norman E Steenrod. Products of cocycles and extensions of mappings. Annals of Mathematics, pages 290-320, 1947.

[Ste04] Richard Steiner. Omega-categories and chain complexes. Homology, Homotopy and Applications, 6(1):175-200, 2004.

[Ste07] Richard Steiner. Orientals. In Categories in algebra, geometry and mathematical physics, volume 431 of Contemp. Math., pages 427-439. Amer. Math. Soc., Providence, RI, 2007.

[Str87] Ross Street. The algebra of oriented simplexes. Journal of Pure and Applied Algebra, 49(3):283-335, 1987.

[Str91] Ross Street. Parity complexes. Cahiers de topologie et géométrie différentielle catégoriques, 32(4):315-343, 1991.

E-mail address: amedinam@nd.edu

Mathematics Department, University of Notre Dame, 255 Hurley, Notre Dame, IN 46556, USA 